University of Texas at El Paso

ScholarWorks@UTEP

$8-2020$

\title{
Two Runners in the Time of Social Distancing, Speedboats in the Gulf of Finland: How to Best Pass?
}

Julio Urenda

The University of Texas at El Paso, jcurenda@utep.edu

Olga Kosheleva

The University of Texas at El Paso, olgak@utep.edu

Vladik Kreinovich

The University of Texas at El Paso, vladik@utep.edu

Follow this and additional works at: https://scholarworks.utep.edu/cs_techrep

Part of the Applied Mathematics Commons, and the Mathematics Commons

Comments:

Technical Report: UTEP-CS-20-90

Published in International Mathematical Forum, 2020, Vol. 15, No. 7, pp. 317-323.

\section{Recommended Citation}

Urenda, Julio; Kosheleva, Olga; and Kreinovich, Vladik, "Two Runners in the Time of Social Distancing, Speedboats in the Gulf of Finland: How to Best Pass?" (2020). Departmental Technical Reports (CS). 1497.

https://scholarworks.utep.edu/cs_techrep/1497

This Article is brought to you for free and open access by the Computer Science at ScholarWorks@UTEP. It has been accepted for inclusion in Departmental Technical Reports (CS) by an authorized administrator of ScholarWorks@UTEP. For more information, please contact Iweber@utep.edu. 


\title{
Two Runners in the Time of Social Distancing, Speedboats in the Gulf of Finland: How to Best Pass?
}

\author{
Julio Urenda ${ }^{1,2}$, Olga Kosheleva ${ }^{3}$, and Vladik Kreinovich ${ }^{2}$ \\ ${ }^{1}$ Department of Mathematical Sciences \\ ${ }^{2}$ Department of Computer Science \\ ${ }^{3}$ Department of Teacher Education \\ University of Texas at El Paso \\ $500 \mathrm{~W}$. University \\ El Paso, TX 79968, USA \\ jcurenda@utep.edu, olgak@utep.edu,vladik@utep.edu
}

\begin{abstract}
If two runners follow the same running path, what is the best trajectory for the faster runner to pass the slower one, taking into account that they should always maintain a prescribed social distance? If a speedboat wants to pass a slower ship following a special canal in the Gulf of Finland, what is the best trajectory? In this paper, we provide answers to both questions.
\end{abstract}

\section{Formulation of the Problem}

Two runners in the time of social distancing: general setting. The need for social distancing, i.e., for every two people to be at least at a distance $d$ from each other ( $d \approx 2$ meters in most countries) has led to many interesting mathematical problems, which can be both useful by themselves and, hopefully, good as entertaining problems for students.

In this paper, we concentrate on a problems related to two runners running along the same running path. We assume that there is a nice running path, and that two runners are exercising along this path.

Need to pass. In practice, the runners' speeds are somewhat different. Without losing generality, let us assume that the second runner is faster. So, if the second runner starts later, then, at some point, this runner has to pass the first one.

What is the best way to do it?

Which way is best. Running along the specially designed path is fun, running nearby is not so pleasant. So, a natural idea is to minimize unpleasantness, i.e., 
to minimize the the length of the trajectory that the passing runner has to follow outside the original path.

In this paper, we provide the desired shortest passing trajectory.

Speedboats in the Gulf of Finland. The above is rather a toy problem, but a similar problem occurs in more practical situations as well, for example, in the Gulf of Finland near St. Petersburg, Russia, where two of the authors (OK and VK) came from.

The gulf is very shallow, especially near St. Petersburg, so to enable big ships to come to the city, a special canal was dug. Smaller boats can also go outside the canal, but it is definitely more convenient for a boat to be in the canal, where there is no need to constantly monitor the depth. So, when a faster boat passes a ship, it tries to minimize the length of its trajectory outside the canal. In this case, the minimal distance $d$ is determined by safety regulations.

Since the problem is similar, our formulas apply to this problem as well.

Comments.

- For simplicity, we will explain all our formulas on the runners example, but these explanation are also valid for a speedboat passing a slower ship traveling through the canal.

- A similar - but simpler - problem was considered in [1].

\section{Simplest Case - When the First Runner Is Very Slow}

Description of the case. Let us first consider the case when the first runner is very slow, i.e., in effect, stays at place.

Analysis of the problem. The second runner has to always be at distance at least $d$ from the first runner, i.e., outside the circle or radius $d$ centered at the location of the first runner.

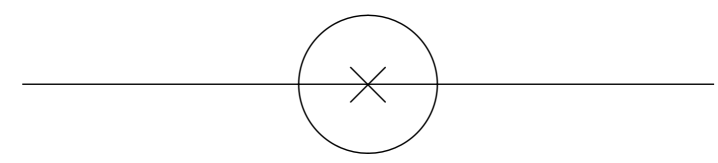

The overall length of the trajectory outside the running path is the smallest if the second runner follows exactly this circle, either to the left or to the right of the running path: 


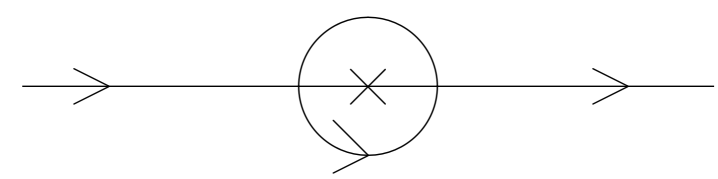

Simplest case: conclusion. The passing runner should wait until the distance $d$ from the first one. Then he/she should follow the circular path - always keeping the distance $d$ from the first runner. This path will return the second runner back to the running path at distance $d$ ahead of the first runner.

\section{General Case}

General case: a brief description. In the general case, the second runner should also keep at the distance $d$ from the first runner - this minimizes the length of the passing runner's outside-running-path trajectory.

Comment. The main difference from the above simplest case is that since the first runner is not staying in place. Because of this, the resulting trajectory of the passing runner will no longer be the circle, it will be more complicated.

General case: analysis of the problem. Let us select the $x$-axis following the running path, in the direction of the runners, and let the $y$-xis be orthogonal to the path.

Let $v_{2}$ be the speed of the second runner, and let $v_{1}<v_{2}$ be the speed of the first runner. For simplicity of calculations, let us select, as the starting moment of time, the moment of time when the second runner appeared $d$ meters behind the first runner - i.e., the moment at which the need to keep social distancing requires the second runner to move away from the path. Similarly, as the starting point for the $x$-axis, let us select the location of the first runner at this moment 0 .

The first runner continues to follow the running path, so the first runner's coordinates $s_{1}(t)=\left(x_{1}(t), y_{1}(t)\right)$ at time $t$ are

$$
s_{1}(t)=\left(v_{1} \cdot t, 0\right) .
$$

Let us denote the coordinates of the second runner by $d_{2}(t)=\left(x_{2}(t), y_{2}(t)\right)$. At moment 0 , the second runner is exactly $d$ meters behind the first one, so

$$
s_{2}(0)=(-d, 0) \text {. }
$$

In terms of these notations, the condition that the distance between the two runners be equal to $d$ during the whole passing process takes the following form:

$$
(x(t))^{2}+\left(y_{2}(t)\right)^{2}=d^{2},
$$


where we denoted

$$
x(t) \stackrel{\text { def }}{=} x_{2}(t)-v \cdot t .
$$

Similarly, the condition that the speed of the second runner is $v_{2}$ takes the form

$$
\left(\dot{x}_{2}(t)\right)^{2}+\left(\dot{y}_{2}(t)\right)^{2}=\left(v_{2}\right)^{2} .
$$

From (4), we conclude that

$$
\dot{x}(t)=\dot{x}_{2}(t)-v,
$$

hence

$$
\dot{x}_{2}(t)=\dot{x}(t)+v \text {. }
$$

Substituting the expression (7) into the formula (5), we conclude that

$$
(\dot{x}(t)+v)^{2}+\left(\dot{y}_{2}(t)\right)^{2}=\left(v_{2}\right)^{2} .
$$

Now, we have a differential equation (8) with two unknown functions: $x(t)$ and $y_{2}(t)$. Let us reduce it to a simpler form, in which we only have one known function $x(t)$. This can be done, since equation (3) enables us to describe $y_{2}$ in terms of $x$ :

$$
\left(y_{2}(t)\right)^{2}=d^{2}-(x(t))^{2} .
$$

Differentiating this expression with respect to time, we conclude that

$$
2 y_{2} \cdot \dot{y}_{2}=-2 x \cdot \dot{x}
$$

Thus,

$$
\dot{y}_{2}=-\frac{x}{y_{2}} \cdot \dot{x}
$$

hence

$$
\left(\dot{y}_{2}\right)^{2}=\frac{x^{2}}{\left(y_{2}\right)^{2}} \cdot(\dot{x})^{2} .
$$

Substituting the expression (9) for $\left(y_{2}\right)^{2}$ into this formula, we conclude that

$$
\left(\dot{y}_{2}\right)^{2}=\frac{x^{2}}{d^{2}-x^{2}} \cdot(\dot{x})^{2} .
$$

Substituting the expression (13) into the formula (8), we conclude that

$$
(\dot{x}+v)^{2}+\frac{x^{2}}{d^{2}-x^{2}} \cdot(\dot{x})^{2}=\left(v_{2}\right)^{2} .
$$

Opening parentheses, moving the term $\left(v_{2}\right)^{2}$ to the left-hand side, and combining terms proportional to 1 , to $\dot{x}$, and to $(\dot{x})^{2}$, we get a quadratic equation for determining $\dot{x}$ :

$$
a \cdot(\dot{x})^{2}+b \cdot \dot{x}+c=0,
$$


where

$$
\begin{gathered}
a=1+\frac{x^{2}}{d^{2}-x^{2}}=\frac{d^{2}}{d^{2}-x^{2}}, \\
b=2 v,
\end{gathered}
$$

and

$$
c=\left(v_{1}\right)^{2}-\left(v_{2}\right)^{2} .
$$

The solution to a quadratic equation has the form

$$
\dot{x}=\frac{-b \pm \sqrt{b^{2}-4 a \cdot c}}{2 a} .
$$

Here, $b<0$, and the passing runner has to move forward $\dot{x}>0$, so we must have the plus sign in the formula (19). Substituting the expressions (15)-(17) into the formula (10), we get

$$
\dot{x}=\frac{-2 v+\sqrt{4\left(v_{1}\right)^{2}+4 \cdot \frac{d^{2} \cdot\left(\left(v_{2}\right)^{2}-\left(v_{1}\right)^{2}\right)}{d^{2}-x^{2}}}}{2 \cdot \frac{d^{2}}{d^{2}-x^{2}}} .
$$

We can divide both the numerator and the denominator by 2, thus getting

$$
\dot{x}=\frac{-v+\sqrt{\left(v_{1}\right)^{2}+\frac{d^{2} \cdot\left(\left(v_{2}\right)^{2}-\left(v_{1}\right)^{2}\right)}{d^{2}-x^{2}}}}{\frac{d^{2}}{d^{2}-x^{2}}} .
$$

In the expression under the square root has the form, we can explicitly add the two expressions, getting:

$$
\begin{aligned}
\left(v_{1}\right)^{2}+\frac{\left.d^{2} \cdot\left(\left(v_{2}\right)^{2}-\left(v_{1}\right)^{2}\right)\right)}{d^{2}-x^{2}} & =\frac{d^{2} \cdot\left(v_{1}\right)^{2}-x^{2} \cdot\left(v_{1}\right)^{2}+d^{2} \cdot\left(v_{2}\right)^{2}-d^{2} \cdot\left(v_{1}\right)^{2}}{d^{2}-x^{2}}= \\
& \frac{d^{2} \cdot\left(v_{2}\right)^{2}-x^{2} \cdot\left(v_{1}\right)^{2}}{d^{2}-x^{2}}
\end{aligned}
$$

so the formula (21) takes the form

$$
\dot{x}=\frac{-v+\sqrt{\frac{d^{2} \cdot\left(v_{2}\right)^{2}-x^{2} \cdot\left(v_{1}\right)^{2}}{d^{2}-x^{2}}}}{\frac{d^{2}}{d^{2}-x^{2}}} .
$$

Multiplying both numerator and denominator by $d^{2}-x^{2}$ and taking into account that for any expression $A$, we have

$$
\sqrt{\frac{A}{d^{2}-x^{2}}} \cdot\left(d^{2}-x^{2}\right)=\sqrt{\frac{A}{d^{2}-x^{2}} \cdot\left(d^{2}-x^{2}\right)^{2}}=\sqrt{A \cdot\left(d^{2}-x^{2}\right)},
$$


we conclude that

$$
\frac{\mathrm{d} x}{\mathrm{~d} t}=\dot{x}=\frac{-v \cdot\left(d^{2}-x^{2}\right)+\sqrt{\left(d^{2} \cdot\left(v_{2}\right)^{2}-x^{2} \cdot\left(v_{1}\right)^{2}\right) \cdot\left(d^{2}-x^{2}\right)}}{d^{2}},
$$

hence

$$
\mathrm{d} t=\frac{d^{2}}{-v \cdot\left(d^{2}-x^{2}\right)+\sqrt{\left(d^{2} \cdot\left(v_{2}\right)^{2}-x^{2} \cdot\left(v_{1}\right)^{2}\right) \cdot\left(d^{2}-x^{2}\right)}} \cdot \mathrm{d} x,
$$

and so, $x(t)$ is determined by the formula

$$
t=\int_{-d}^{x} \frac{d^{2}}{-v \cdot\left(d^{2}-\left(x^{\prime}\right)^{2}\right)+\sqrt{\left(d^{2} \cdot\left(v_{2}\right)^{2}-\left(x^{\prime}\right)^{2} \cdot\left(v_{1}\right)^{2}\right) \cdot\left(d^{2}-\left(x^{\prime}\right)^{2}\right)}} \mathrm{d} x^{\prime} .
$$

Conclusion. When the faster runner, with speed $v_{2}>v_{1}$, approaches the distance $d$ behind the first runner, the faster runner starts passing. To describe the passing, we took the moment of time when passing started at moment 0 , and we took the $x$-axis along the running path.

In these terms, the optimal passing trajectory is as follows: $x_{2}(t)=x(t)+v \cdot t$, where $x(t)$ is determined by the formula (28), and

$$
y_{2}(t)=\sqrt{d^{2}-(x(t))^{2}} .
$$

Comment. The above formulas are complicated, but they are relatively easy to implement: just keep the distance $d$ from the first runner while maintaining your speed $v_{2}$.

\section{Acknowledgments}

This work was supported in part by the National Science Foundation grants 1623190 (A Model of Change for Preparing a New Generation for Professional Practice in Computer Science), HRD-1834620 (CAHSI Includes), and HRD1242122 (Cyber-ShARE Center of Excellence).

\section{References}

[1] J. C. Urenda, O. Kosheleva, M. Ceberio, and V. Kreinovich, "How mathematics and computing can help fight the pandemic: two pedagogical examples", In: Proceedings of the Annual Conference of the North American Fuzzy Information Processing Society NAFIPS'2020, Redmond, Washington, August 20-22, 2020, to appear. 